\title{
APPLICATIONS OF A SET-THEORETIC LEMMA
}

\author{
GARY GRUENHAGE
}

\begin{abstract}
A set-theoretic lemma is introduced and various applications are given, including: (1) a result of Erdös and Hajnal on the coloring number of a graph; (2) game characterizations of the coloring number of a graph; (3) K. Alster's result that a point-countable collection of open, compact scattered spaces has a point-finite clopen refinement; (4) normal, locally compact, metacompact spaces which are scattered of finite height are paracompact.
\end{abstract}

1. Introduction. In this paper, a purely set-theoretic lemma is presented which appears to be the "common part" of $\mathbf{K}$. Alster's result [A] that a point-countable collection of compact scattered spaces has a point-finite clopen (open and closed) refinement, and a result of Erdös and Hajnal $[\mathbf{E H}]$ on the coloring number of a graph. The author has found this lemma to be quite useful (see $\left[\mathbf{G}_{2}\right]$, for example). In this paper, we will show how the lemma can be used to prove the above results, and we will also give some other applications. One such application is a "game characterization" of the coloring number of a graph, from which the Erdös-Hajnal result easily follows. Other applications are partial answers to the question of whether metalindelöf spaces are preserved by closed maps, and Tall's question of whether normal, locally compact, metacompact spaces are paracompact.

2. The lemma. In this section, we state and prove the main lemma. In the lemma, $2^{M}$ is the set of all subsets of $M$, and $A_{\alpha} \nearrow A$ means that, for some ordinal $\kappa$, $\left(A_{\alpha}\right)_{\alpha<\kappa}$ is an increasing sequence of sets whose union is $A$.

LEMMA 2.1. ${ }^{1}$ Let $M$ be a set, and let $\phi: 2^{M} \rightarrow 2^{M}$ satisfy

(1) $|\phi(A)| \leqslant \max (|A|, \omega)$;

(2) $A_{\alpha} \nearrow A \Rightarrow \phi\left(A_{\alpha}\right) \nearrow \phi(A)$.

Call $A \subset M \phi$-closed if $\phi(A) \subset A$. Suppose $\mathrm{P}$ is a property of subsets of $M$. Then $M$ has property $\mathrm{P}$ whenever $\mathrm{P}$ satisfies the following properties:

(i) $\mathrm{P}$ holds for all countable $\phi$-closed sets;

(ii) $\mathrm{P}$ holds for all sets which are increasing unions of $\phi$-closed sets satisfying $\mathrm{P}$.

Proof. The proof is by induction on $|M|$. If $|M| \leqslant \omega$, then $M$ satisfies $\mathrm{P}$ by property (i). Suppose $|M|=\kappa>\omega$, and the lemma holds for all sets of cardinality less than $\kappa$. Let $M=\left\{m_{\alpha}: \alpha<\kappa\right\}$. We inductively construct $\left(M_{\alpha}\right)_{\alpha<\kappa}$ such that, for

Received by the editors August 12, 1983.

1980 Mathematics Subject Classification. Primary 54D18, 04A20.

${ }^{1}$ This lemma and its proof also appears in $\left[\mathbf{G}_{2}\right]$. It is included here for the reader's convenience. 
each $\alpha$,

(a) $M_{\alpha} \supset\left\{m_{\beta}: \beta<\alpha\right\}$

(b) $\left|M_{\alpha}\right| \leqslant \max \{|\alpha|, \omega\}$;

(c) $M_{\alpha}$ is $\phi$-closed.

Let $M_{0}=\varnothing$. Suppose $\alpha<\kappa$, and $M_{\beta}$ has been defined for all $\beta<\alpha$. If $\alpha$ is a limit ordinal, let $M_{\alpha}=\bigcup_{\beta<\alpha} M_{\beta}$. If $\alpha=\alpha^{\prime}+1$, let $M_{\alpha, 0}=M_{\alpha^{\prime}} \cup\left\{m_{\alpha^{\prime}}\right\}$, and let

$$
M_{\alpha, n+1}=M_{\alpha, n} \cup \phi\left(M_{\alpha, n}\right) \text {. }
$$

Then let $M_{\alpha}=\bigcup_{n \in \omega} M_{\alpha, n}$. It is easily checked that (a)-(c) hold. (Property (c) follows from property (2) in the statement of the lemma.)

Now each $M_{\alpha}$ and $\phi \mid 2^{M_{\alpha}}$ satisfy the conditions of the lemma. Hence each $M_{\alpha}$ satisfies $\mathrm{P}$, and so by property (ii) of $\mathrm{P}$, we conclude that $M$ satisfies $\mathrm{P}$.

REMARK. It is easy to see from 2.1(2) that if $A_{\alpha} \nearrow A$ and each $A_{\alpha}$ is $\phi$-closed, then $A$ is also $\phi$-closed. This fact will often be used in the sequel.

3. The coloring number of a graph. Let $G=(V, E)$ be a graph with vertices $V$ and edges $E$. The chromatic number of $G$, denoted $\operatorname{chr}(G)$, is the least cardinal $\kappa$ such that the vertices can be colored with $\kappa$ colors so that adjacent vertices get different colors. The coloring number of $G$, denoted $\operatorname{col}(G)$, is the least cardinal $\lambda$ such that there exists a well-order $\leqslant$ on $V$ with $\mid\{y<x: y$ is adjacent to $x\} \mid<\lambda$ for each $x \in V$.

If $\operatorname{col}(G) \leqslant \kappa$, it is easy to use the well-order to show that $\operatorname{chr}(G) \leqslant \kappa$; hence $\operatorname{chr}(G) \leqslant \operatorname{col}(G)$. This inequality is often strict; e.g., the graph $K\left(\omega, \omega_{1}\right)$ defined in the next paragraph has coloring number $\omega_{1}$ and chromatic number 2 .

For cardinals $\kappa$ and $\lambda$, let $K(\kappa, \lambda)$ be a graph with vertices $V=A \cup B$, where $|A|=\kappa$ and $|B|=\lambda, A \cap B=\varnothing$, and $E=\{\{a, b\}: a \in A, b \in B\}$. Erdös and Hajnal [EH, Corollary 5.6] proved the following result.

THEOREM 3.1. If $\operatorname{col}(G)>\omega$, then $G$ contains a copy of $K\left(n, \omega_{1}\right)$ for each $n \in \omega$.

Proof. Assume $G$ does not contain $K\left(n, \omega_{1}\right)$ for some fixed $n \in \omega$. We show $\operatorname{col}(G) \leqslant \omega$. For $H \subset V$, define

$$
\Phi(H)=\{x \in V: x \text { is adjacent to } \geqslant n \text { elements of } H\} .
$$

Since $G \not \supset K\left(n, \omega_{1}\right)$, only countably many vertices are adjacent to each vertex of any particular set of $n$ distinct vertices. Then it is easy to see that $\Phi$ satisfies (1) and (2) of Lemma 2.1 (with $M=V$ ).

For $H \subset V$, let $\mathrm{P}(H)$ be the property that the vertices of $H$ can be well-ordered as desired (i.e., $\{y<x: y$ is adjacent to $x\}$ is finite for each $x \in H$ ). Clearly, $\mathrm{P}(H)$ holds for all countable $H$ (simply enumerate $H$ ), so $\mathrm{P}$ satisfies 2.1(i). It remains to prove that P satisfies 2.1(ii). Suppose $H_{\alpha} \nearrow H, \alpha<\kappa$, where each $H_{\alpha}$ is $\Phi$-closed and satisfies P. Let $\leqslant$ be the well-order of $H_{\alpha}$ with our desired properties. For $x \in H$, let $\alpha(x)$ be the least $\alpha$ with $x \in H_{\alpha}$. If $x, y \in H$, define $x<y$ iff

(a) $\alpha(x) \lesseqgtr \alpha(y)$, or

(b) $\alpha(x)=\alpha(y)=\alpha$, and $x \leqslant_{\alpha} y$.

By a standard argument, this defines a well-order on $H$. 
Now suppose $x \in H$; we wish to prove that there are only finitely many $y \leqslant x$ with $y$ adjacent to $x$. Since $\bigcup_{\beta<\alpha(x)} H_{\beta}$ is $\Phi$-closed, $x$ is adjacent to at most $n-1 y$ 's with $\alpha(y) \supsetneqq \alpha(x)$. And by definition of $\leqslant_{\alpha}$, there are only finitely many $z$ adjacent to $x$ with $\alpha(z)=\alpha(x)$ and $z \leqslant \leqslant_{(x)} x$. Thus " $\leqslant$ " has the desired properties.

In much the same spirit, we can prove some "game characterizations" of the statement " $\operatorname{col}(G) \leqslant \omega$ " from which the previous theorem can be easily derived. The following formulation of these games was suggested by F. Galvin, which greatly improved an earlier version due to this author.

Let $G=(V, E)$ be a nonempty graph. We consider the following games of length $\omega$ with players "Black" and "White". At the $n$th play, first Black chooses $B_{n} \subset V$ such that (choose one):

$$
\left\{\begin{array}{l}
\left|B_{n}\right|=1, \\
1 \leqslant\left|B_{n}\right|<\boldsymbol{\aleph}_{0}, \\
1 \leqslant\left|B_{n}\right| \leqslant \boldsymbol{\aleph}_{0} .
\end{array}\right.
$$

Then White chooses $W_{n} \subset V$ such that (choose one):

$$
\left\{\begin{array}{l}
\left|W_{n}\right|=1 \\
\left|W_{n}\right|<\aleph_{0} \\
\left|W_{n}\right| \leqslant \aleph_{0} .
\end{array}\right.
$$

Let $B=\bigcup_{n \in \omega} B_{n}$ and $W=\bigcup_{n \in \omega} W_{n}$. Black wins if and only if $B$ is infinite, and, there is a vertex $v \notin W$ which is adjacent to (choose one):

$$
\left\{\begin{array}{l}
\text { infinitely many, } \\
\text { all }
\end{array}\right.
$$

elements of $B$.

To illustrate a situation in which White has a winning strategy, suppose $n \in \omega$ is such that $G$ does not contain a copy of $K\left(n, \omega_{1}\right)$. Then it is easy for White to make sure that $W$ contains all vertices adjacent to $n$ or more elements of $B$. In this case, White wins. Thus Theorem 3.1 is an easy corollary of

THEOREM 3.2. Let $G$ be a graph and let $\Gamma(G)$ be one of the games defined above. Then $\operatorname{col}(G) \leqslant \omega$ if and only if White has a winning strategy in $\Gamma(G)$.

Proof. Suppose the well-order $\leqslant$ on $G$ witnesses $\operatorname{col}(G) \leqslant \omega$. Then White can easily make sure that $W$ contains all vertices $y$ adjacent to some $b \in B$ with $y \leqslant b$. Clearly White wins in this case.

Now suppose White has a winning strategy $\sigma$ in $\Gamma(G)$. This means that if White plays $\sigma\left(\left\langle B_{0}, W_{0}, \ldots, B_{n}\right\rangle\right)$ whenever $\left\langle B_{0}, W_{0}, \ldots, B_{n}\right\rangle$ has been the sequence of plays by Black and White, then White wins. For $H \subset V$, let $\Phi(H)$ be the union of all sets $\sigma\left(\left\langle B_{0}, W_{0}, \ldots, B_{n}\right\rangle\right)$, where $B_{i} \subset H$ and $\left|B_{i}\right|<\omega$ for each $i$, and $W_{i}=$ $\sigma\left(\left\langle B_{0}, W_{0}, \ldots, W_{i-1}, B_{i}\right\rangle\right)$ for each $i=0,1, \ldots, n-1$. It is easy to check that $\Phi$ satisfies (1) and (2) of Lemma 2.1. 
The rest of the proof is the same as that of Theorem 3.1, except the statement " $x$ is adjacent to only finitely many $y$ with $\alpha(y) \supsetneqq \alpha(x)$ " needs slightly more argument, as follows. Suppose $y_{0}, y_{1}, \ldots$ are distinct elements of $\cup_{\beta<\alpha(x)} H_{\beta}$ with each $y_{n}$ adjacent to $x$. Black could choose $B_{n}=\left\{y_{n}\right\}$, hence $B=\left\{y_{n}: n \in \omega\right\}$. Since $\bigcup_{\beta<\alpha(x)} H_{\beta}$ is $\Phi$-closed, it follows that $x \notin W$. But then Black wins, a contradiction.

4. Some applications to topology. The topological applications we present involve locally compact or local Lindelöf spaces. The first is a result of K. Alster. This was the key result in his proof that Corson compact scattered spaces are strong Eberlein compact $[\mathbf{A}]$.

Recall that a space $X$ is scattered if it contains no dense-in-itself subsets. If we let $X^{(0)}$ be the isolated points of $X$, and $X^{(\alpha)}$ the isolated points of $X-\cup_{\beta<\alpha} X^{(\beta)}$, then the least ordinal $\gamma$ such that $X^{(\gamma)}=\varnothing$ is called the scattering height of $X$. Observe that if $X$ is a compact scattered space of height $\gamma$, then the "top level" $X^{(\gamma-1)}$ of $X$ is finite (being closed discrete in $X$ ). In the sequel, we will let $T(X)$ denote this top level of the compact scattered space $X$.

THEOREM 4.1 (ALSTER). If $\mathscr{U}$ is a point-countable collection of compact scattered open subsets of a space $X$, then $\mathscr{U}$ has a point-finite clopen refinement.

Proof. We apply Lemma 2.1 , with $M=\mathscr{U}$. For $\mathscr{V} \subset \mathscr{U}$, let

$$
T(\mathscr{V})=\bigcup\{T(Z): Z=\cap \mathscr{F}, \mathscr{F} \text { a finite subset of } \mathscr{V}\}
$$

and

$$
\Phi(\mathscr{V})=\{U \in \mathscr{U}: U \cap T(\mathscr{V}) \neq \varnothing\}
$$

It is routine to check that $\Phi$ satisfies (1) and (2) of Lemma 2.1.

Let $\mathrm{P}(\mathscr{V})$ be the property that $\mathscr{V}$ has a point-finite clopen refinement. Then $\mathrm{P}(\mathscr{V})$ holds for countable $\mathscr{V}$ since $\cup \mathscr{V}$ is $\sigma$-compact. Suppose $\mathscr{V}_{\alpha} \nearrow \mathscr{V}, \alpha<\kappa$, where $\mathscr{V}_{\alpha}$ is $\Phi$-closed and satisfies P. To complete the proof, we need to show that $\mathscr{V}$ has a point-finite clopen refinement. Now each $\mathscr{V}_{\alpha}$ has a point-finite clopen refinement $W_{\alpha}$. Let

$$
\mathscr{W}_{\alpha}^{\prime}=\left\{W \in \mathscr{W}_{\alpha}: W \not \subset \bigcup_{\beta<\alpha}\left(\bigcup \mathscr{V}_{\beta}\right)\right\}
$$

and let $\mathscr{W}^{\prime}=U_{\alpha<\kappa} \mathscr{W}_{\alpha}^{\prime}$. Then $\mathscr{W}^{\prime}$ is a refinement of $\mathscr{V}$. It remains to prove that $\mathscr{W}^{\prime}$ is point-finite.

If $\mathscr{W}^{\prime}$ is not point-finite, then there exists $x \in X$ and $W_{\alpha(n)} \in \mathscr{W}_{\alpha(n)}^{\prime}, n \in \omega$, with $x \in \bigcap_{n \in \omega} W_{\alpha(n)}$. Since each $\mathscr{W}_{\alpha}$ is point-finite, we can assume that $(\alpha(n))_{n \in \omega}$ is a strictly increasing sequence of distinct ordinals. Let $W_{\alpha(n)} \subset V_{n} \in \mathscr{V}_{\alpha(n)}$. If $\gamma_{k}$ is the height of the scattered space $\bigcap_{i \leqslant k} V_{i}$, then $\left(\gamma_{k}\right)_{k \in \omega}$ is a nonincreasing sequence of ordinals, so $\gamma_{m}=\gamma_{m+1}=\cdots$ for some $m \in \omega$. Then $V_{m+1} \cap T\left(\cap_{i \leqslant m} V_{i}\right) \neq \varnothing$. But then if $\hat{\mathscr{V}}=\bigcup_{\beta<\alpha(m+1)} \mathscr{V}_{\beta}$, we have $V_{m+1} \in \hat{\mathscr{V}}$, since $\hat{\mathscr{V}}$ is $\Phi$-closed and contains each $V_{i}, i \leqslant m$. This contradicts $W_{\alpha(m+1)} \subset V_{m+1}$ and $W_{\alpha(m+1)} \not \subset U_{\beta<\alpha(m+1)}\left(\cup \mathscr{V}_{\beta}\right)$. 
We mention without proof another application which appears in $\left[\mathbf{G}_{\mathbf{2}}\right]$. Call a closed subset $H$ of a space $X$ a $W$-set in $X$ if Player I has a winning strategy in the following 2-person infinite game. At the $n$th play, Player I chooses an open set $U_{n} \supset H$, and Player II chooses $x_{n} \in U_{n}$. Player I wins if $x_{n} \rightarrow H$, i.e., every neighborhood of $H$ contains all but finitely many $x_{n}$.

THEOREM 4.2. (a) If $X$ is a compact space having countable tightness, ${ }^{2}$ then a closed subset $H$ of $X$ is $a W$-set in $X$ if and only if $X-H$ is metalindelöf.

(b) If $X$ is a compact scattered space, then a closed subset $H$ of $X$ is a $W$-set in $X$ if and only if $X-H$ is metacompact.

The proof of 4.2 is quite similar to a combination of the proofs of Alster's theorem and the game characterization of $\operatorname{col}(G) \leqslant \omega$ given in $\S 3$. See $\left[\mathbf{G}_{2}\right]$ for the details.

As another application of Lemma 2.1, we give a partial solution to the following question of $\mathrm{F}$. Tall: Is every normal, locally compact, metacompact space $X$ paracompact? A number of partial solutions have already been obtained. In particular, the answer is known to be positive in the following situations:

(a) $X$ is perfectly normal (Arhangel'skii [Ar]);

(b) $X$ is locally CCC (Tall [ $\left.\left.\mathbf{T}_{2}\right]\right)$;

(c) $X$ is locally connected (Gruenhage $\left[\mathbf{G}_{1}\right]$ );

(d) $V=L$ (Watson [W]);

(e) $X$ is boundedly metacompact (Daniels [D]).

A cover $\mathscr{U}$ of $X$ is boundedly point-finite if there exists $n \in \omega$ such that each point of $X$ is in at most $n$ elements of $\mathscr{U}$. A space $X$ is boundedly metacompact if each open cover of $X$ has a boundedly point-finite open refinement.

Daniels showed something slightly stronger than (e), namely, that a normal space having a boundedly point-finite cover by open sets with compact closures is paracompact. She also showed that if there is a zero-dimensional counterexample to Tall's question, then there is one which is a subspace of the Pixley-Roy space over some cardinal $\kappa$, where $\kappa$ carries the cofinite topology. Since these Pixley-Roy spaces are scattered, we have that a zero-dimensional counterexample implies the existence of a scattered one. ${ }^{3}$ The following result eliminates the simplest type of scattered spaces as possible counterexamples.

THEOREM 4.3. If $X$ is normal, locally compact, metacompact, and scattered of finite height, then $X$ is paracompact. ${ }^{4}$

Proof. The proof is by induction on the scattering height. Assume $X$ is a normal, locally compact scattered space of height $n+1$, and the theorem is true for spaces of smaller height. To prove that $X$ is paracompact, we need only prove that the discrete collection $X^{(n)}$ of points of the top level can be separated by a disjoint collection

\footnotetext{
${ }^{2} \mathrm{~A}$ space $X$ has countable tightness if $x \in \bar{A}$ implies $x \in \bar{C}$ for some countable $C \subset A$.

${ }^{3}$ Perhaps (c) above can be taken as evidence that if there is a counterexample at all then there is a 0-dimensional one.

${ }^{4} \mathrm{E}$. van Douwen has observed that this result holds for spaces of height $\omega$ as well-just use countable paracompactness to reduce it to the finite height case.
} 
$\mathcal{O}=\left\{O_{x}: x \in X^{(n)}\right\}$ of open sets-for then by normality and zero-dimensionality we can assume $\mathcal{O}$ is a discrete collection of compact open sets and so $\cup \mathcal{O}$ and $X-\cup \mathcal{O}$ partitions $X$ into two clopen paracompact pieces.

By the assumptions, there exists a point-finite collection $\mathscr{U}=\left\{U_{x}: x \in X^{(n)}\right\}$ of compact open subsets of $X$ with $U_{x} \cap X^{(n)}=\{x\}$ for each $x \in X^{(n)}$. As before, let $T(Z)$ denote the finite set of points of the top level of the compact scattered space $Z$. For $A \subset X^{(n)}$, let

$$
\Phi(A)=\left\{x \in X^{(n)}: U_{x} \cap T(\cap \mathscr{F}) \neq \varnothing \text { for some finite } \mathscr{F} \subset\left\{U_{x}: x \in A\right\}\right\} .
$$

It is easy to check that $\Phi$ satisfies the conditions of Lemma 2.1 with $M=X^{(n)}$.

Let $\mathrm{P}(A)$ be the property that $A$ can be separated. Then $\mathrm{P}$ holds for all countable $A \subset X^{(n)}$. Suppose $A_{\alpha} \nearrow A, \alpha<\kappa$, where each $A_{\alpha}$ is $\Phi$-closed and satisfies P. For each $\alpha<\kappa$, let $\left\{U_{x, \alpha}: x \in A_{\alpha}\right\}$ be a discrete collection of compact open sets separating $A_{\alpha}$ such that $x \in U_{x, \alpha} \subset U_{x}$.

Now let $\mathscr{V}=\left\{U_{x, \alpha(x)}: x \in A\right\}$, where $\alpha(x)$ is the least $\alpha$ with $x \in A_{\alpha}$. The key idea here is to show that $\mathscr{V}$ is boundedly point-finite, and hence Daniel's theorem can be used.

We show that each $y \in X$ is in at most $n+1$ elements of $\mathscr{V}$. If not, there exist $x_{i} \in A$ with $y \in \bigcap_{i=0}^{n+1} U_{x_{i}, \alpha\left(x_{i}\right)}$, and $\alpha\left(x_{0}\right)<\alpha\left(x_{1}\right)<\cdots<\alpha\left(x_{n+1}\right)$. Then for each $j \leqslant n+1$, we have $U_{x_{j}} \cap T\left(\cap_{i<j} U_{x_{i}}\right)=\varnothing$ (otherwise, $x_{j} \in A_{a\left(x_{j-1}\right)}$ by " $\Phi$-closedness"). Hence if $\gamma_{j}$ is the height of $\bigcap_{i \leqslant j} U_{x_{i}}$, then $\gamma_{0}>\gamma_{1}>\cdots>\gamma_{n+1}$, contradicting $\gamma_{0}<n+1$ and $\gamma_{n+1} \geqslant 1$.

Now to complete the proof that $A$ can be separated, use normality to obtain an open set $W$ such that $A \subset W \subset \bar{W} \subset \cup \mathscr{V}$. Then $\bar{W}$ has a boundedly point-finite cover by compact open sets, so by Daniels' result, $\bar{W}$ is paracompact. It follows that $A$ can be separated in $\bar{W}$, hence in $X$.

Our last "application" can be considered an application of a much weaker form of Lemma 2.1 in which property $P$ satisfies

(ii)' If $A$ is the union of $\Phi$-closed sets satisfying $\mathrm{P}$, then $A$ satisfies $\mathrm{P}$.

In this case the proof of Lemma 2.1 is very simple. No induction is needed: one easily shows that $M$ is a union of countable $\Phi$-closed sets, and hence $M$ satisfies P. So the proof of this modified version of 2.1 is easily incorporated into the proof of any result one may wish to apply it to. Hence I think of this type of "application" more as an application of the idea of using $\Phi$-closed collections than of the lemma. The result below illustrates one such application; see [GM] for another.

Let us recall that it is an open question whether metalindelöf ${ }^{5}$ spaces are preserved by closed or perfect maps $\left(\right.$ see $\left.\left[\mathrm{Bu}_{1}\right]\right)$. It is known that hereditarily metalindelöf spaces, as well as $\omega_{1}$-Lindelöf ${ }^{6}$ metalindelöf spaces, are so preserved (see [ $\mathbf{B u}_{2}$ and $\left.\left.\mathbf{G M T}\right]\right)$. The following is another partial answer to this question.

THEOREM 4.4. Let $X$ be a regular metalindelöf, locally Lindelöf space, and let $f$ : $X \rightarrow Y$ be a closed map with Lindelöf fibers. Then $Y$ is metalindelöf.

\footnotetext{
${ }^{5} \mathrm{~A}$ space $X$ is metalindelöf if every open cover of $X$ has a point-countable open refinement.

${ }^{6} \mathrm{~A}$ space $X$ is $\omega_{1}$-Lindelöf if every open cover of $X$ has a subcover of cardinality $\leqslant \omega_{1}$.
} 
Proof. We first claim that each $y \in Y$ has a neighborhood $L_{y}$ such that $f^{-1}\left(\bar{L}_{y}\right)$ is Lindelöf. To see this, let $\left\{U_{n}: n \in \omega\right\}$ be a cover of $f^{-1}(y)$ by open sets with Lindelöf closures. Let $L_{y}^{\prime}$ be a neighborhood of $y$ such that $f^{-1}\left(L_{y}^{\prime}\right) \subset \bigcup_{n \in \omega} U_{n}$. Now let $L_{y}$ be a neighborhood of $y$ such that $\bar{L}_{y} \subset L_{y}^{\prime}$. Then $f^{-1}\left(\bar{L}_{y}\right)$ is a closed subspace of the Lindelöf space $\bigcup_{n \in \omega} \bar{U}_{n}$. Hence $f^{-1}\left(\bar{L}_{y}\right)$ is Lindelöf, so $\vec{L}_{y}$ is, too.

Let $\mathscr{L}$ be an open cover of $Y$ such that $f^{-1}(\bar{L})$ is Lindelöf for each $L \in \mathscr{L}$. Let $\mathscr{U}$ be a point-countable open refinement of $f^{-1}(\mathscr{L})=\left\{f^{-1}(L): L \in \mathscr{L}\right\}$. Note that each $V \in \mathscr{U}$ has the property that $f^{-1}(f(\bar{V}))$ is Lindelöf; hence there exists a countable $\mathscr{U}(V) \subset \mathscr{U}$ which covers $f^{-1}(f(\bar{V}))$. Now for $\mathscr{V} \subset \mathscr{U}$, let

$$
\Phi(\mathscr{V})=\bigcup\{\mathscr{U}(V): V \in \mathscr{V}\}
$$

Then " $\mathscr{V}$ is $\Phi$-closed" implies that $\mathscr{V}$ contains a cover of $f^{-1}(f(\bar{V}))$ for each $V \in \mathscr{V}$, hence $f^{-1}(f(\cup \mathscr{V}))=\cup \mathscr{V}$, and so $f(\cup \mathscr{V})$ is open.

One easily sees that $\mathscr{U}$ can be written as $\bigcup_{\alpha<\kappa} \mathscr{U}_{\alpha}$, where each $\mathscr{U}_{\alpha}$ is $\Phi$-closed and countable. Then $\cup \mathscr{U}_{\alpha}$ is Lindelöf, and so $f\left(\cup \mathscr{U}_{\alpha}\right)$ is an open Lindelöf subset of $Y$. Now let $\mathscr{U}_{\alpha}^{\prime}=\mathscr{U}_{\alpha}-\cup_{\beta<\alpha} \mathscr{U}_{\beta}$, and let

$$
W_{\alpha}=\left\{y \in Y: f^{-1}(y) \subset \cup \mathscr{U}_{\alpha}^{\prime}\right\}
$$

Claim. $\mathscr{W}=\left\{W_{\alpha}: \alpha<\kappa\right\}$ is a point-countable open cover of $Y$.

That $W_{\alpha}$ is open follows from the fact that $f$ is closed. To see that $\mathscr{W}$ covers $Y$, suppose $y \in Y$ and let $\alpha(y)$ be the least $\alpha$ with $f^{-1}(y) \cap\left(\cup \mathscr{U}_{\alpha}\right) \neq \varnothing$. Since $\mathscr{U}_{\alpha}$ is $\Phi$-closed, it follows that $f^{-1}(y) \subset \cup \mathscr{U}_{\alpha}$, and so $f^{-1}(y) \subset \cup \mathscr{U}_{\alpha}^{\prime}$. Thus $\mathscr{W}$ is a cover of $Y$. Finally, we check that $\mathscr{W}$ is point-countable. Pick $y \in Y$ and $x \in f^{-1}(y)$, and for each $\alpha$ with $y \in W_{\alpha}$, pick $U_{\alpha} \in \mathscr{U}_{\alpha}^{\prime}$ with $x \in U_{\alpha}$. Since the $U_{\alpha}$ 's must be distinct and since $\mathscr{U}$ is point-countable, it follows that the set $\left\{\alpha: y \in W_{\alpha}\right\}$ is countable. This establishes the claim.

To complete the proof, suppose $\mathcal{O}$ is an open cover of $Y$. For each $\alpha$, since $f\left(\cup \mathscr{U}_{\alpha}\right)$ is Lindelöf, we can let $\mathscr{M}_{\alpha}$ be a countable open cover of $f\left(\cup \mathscr{U}_{\alpha}\right)$ refining $\mathcal{O}$. Then $\bigcup_{\alpha<\kappa}\left\{M \cap W_{\alpha}: M \in \mathscr{M}_{\alpha}\right\}$ is a point-countable open refinement of $\mathcal{O}$ which covers $Y$.

\section{REFERENCES}

[A] K. Alster, Some remarks on Eberlein compacts, Fund. Math. 104 (1979), 43-46.

[Ar] A. V. Arhangel'skii, The property of paracompactness in the class of perfectly normal, locally bicompact spaces, Soviet Math. Dokl. 12 (1971), 1253-1257.

[Bu $\left.\mathbf{B u}_{1}\right]$ D. Burke, Closed mappings, Surveys in General Topology (G. M. Reed, ed.), Academic Press, 1980.

$\left[\mathrm{Bu}_{2}\right] \ldots$, Characterizations of meta-Lindelöf and related spaces (preprint).

[D] M. Daniels, Normal locally compact boundedly metacompact spaces are paracompact, Canad. J. Math. 35 (1983), 807-823.

[EH] P. Erdös and A. Hajnal, On chromatic number of graphs and set-systems, Acta Math. Acad. Sci. Hungar. 17 (1966), 61-99.

$\left[\mathbf{G}_{1}\right]$ G. Gruenhage, Paracompactness in normal, locally connected, locally compact spaces, Topology Proc. 4 (1979), 393-405.

$\left[\mathbf{G}_{\mathbf{2}}\right] \_$, Covering properties on $X^{2} \backslash \Delta, W$-sets, and compact subsets of $\Sigma$-products, Topology Appl. (to appear).

[GM] G. Gruenhage and E. Michael, A result on shrinkable open covers, Topology Proc. 8 (1983), 37-43. 
[GMT] G. Gruenhage, E. Michael and Y. Tanaka, Spaces determined by point-countable covers, Pacific J. Math. (to appear).

[ $\left.\mathbf{T}_{1}\right] \mathrm{F}$. Tall, On the existence of normal metacompact Moore spaces which are not metrizable, Canad. J. Math. 26 (1974), 1-6.

$\left[\mathbf{T}_{2}\right]$ _. The countable chain condition versus separability-applications of Martin's Axiom, General Topology Appl. 2 (1974), 315-339.

[W] W. S. Watson, Locally compact normal spaces in the constructible universe, Canad. J. Math. 34 (1982), 1091-1096.

Department of Mathematics, Auburn University, Auburn, Alabama 36830 